\title{
Review: specific combinations of symptoms effectively rule in the diagnosis of urinary tract infection based on history alone
}

Bent S, Nallamothu BK, Simel DL, et al. Does this woman have an acute uncomplicated urinary tract infection? JAMA

2002 May 22-29;287:2701-10.

\section{QUESTION: Are history taking and physical examination accurate and precise for diagnosis of acute urinary tract infection (UTI) in women?}

\section{Data sources}

English language studies were identified by searching Medline (1966 to September 2001); contacting experts in the field; and reviewing bibliographies, core articles, and 3 commonly used textbooks.

\section{Study selection}

Studies were selected if they contained original data on the accuracy or precision of history taking or physical examination for diagnosing acute uncomplicated UTI in women. Studies were excluded if they evaluated infants, children or adolescents, pregnant women, or nursing home patients or if there were insufficient data to calculate likelihood ratios (LRs).

\section{Data extraction}

2 reviewers independently extracted data on methodological quality and patient characteristics. Published raw data were used to calculate summary LRs using a random effects model.

\section{Main results}

9 studies met the selection criteria. 8 studies examined the accuracy of 9 different symptoms for predicting the presence of UTI. 4 symptoms and 1 sign increased the probability of UTI (table 1). 4 symptoms and 1 sign decreased the probability of UTI (table 2), of which his-

Table 1. Positive likelihood ratios (+LRs) for symptoms and signs that increased the probability of urinary tract infection in women*

\begin{tabular}{ll} 
Symptom or sign & Summary +LR $(95 \% \mathrm{Cl})$ \\
\hline Dysuria & $1.5(1.2$ to 2.0$)$ \\
\hline Frequency & $1.8(1.1$ to 3.0$)$ \\
\hline Haematuria & $2.0(1.3$ to 2.9$)$ \\
\hline Back pain & $1.6(1.2$ to 2.1 \\
\hline $\begin{array}{l}\text { Costovertebral angle } \\
\text { tenderness }\end{array}$ & $1.7(1.1$ to 2.5$)$ \\
\hline${ }^{*}$ Diagnostic terms defined in glossary. &
\end{tabular}

Table 2. Negative likelihood ratios (-LRs) for symptoms and signs that decreased the probability of urinary tract infection*

\begin{tabular}{ll} 
Symptom or sign & Summary -LR $(95 \% \mathrm{Cl})$ \\
Absence of dysuria & $0.5(0.3$ to 0.7$)$ \\
\hline Absence of back pain & $0.8(0.7$ to 0.9$)$ \\
\hline History of vaginal discharge & $0.3(0.1$ to 0.9$)$ \\
\hline History of vaginal irritation & $0.2(0.1$ to 0.9$)$ \\
\hline $\begin{array}{l}\text { Vaginal discharge on } \\
\text { examination }\end{array}$ & $0.7(0.5$ to 0.9$)$ \\
\hline${ }^{*}$ Diagnostic terms defined in glossary.
\end{tabular}

tory of vaginal discharge and history of vaginal irritation were the most powerful. 1 study examined combinations of symptoms and found that presence of dysuria and frequency without vaginal discharge or irritation was associated with a high positive LR for UTI (24.6). 1 study of patients with recurrent UTI found that self diagnosis increased the probability of UTI (positive LR 4.0).

\section{Conclusions}

Several different symptoms and signs are associated with an increased or decreased probability of urinary tract infection (UTI) in women. Specific combinations of symptoms effectively rule in the diagnosis of UTI based on history alone.

\section{COMMENTARY}

An estimated 5\% of women experience $\geq 1$ UTI annually. ${ }^{1}$ Diagnostic strategies for this common and distressing health condition vary substantially among clinicians. Diagnoses are often based on conventional wisdom passed between generations of providers rather than on rigorously obtained, data based evidence.

The findings of the systematic review by Bent $e t$ al will be useful for many clinicians. Evidence that women with recurrent UTIs may be able to accurately diagnose their UTIs emphasises the importance of listening to patients and valuing their perceptions. Furthermore, recent guidelines advise that the history and physical examination often are sufficient for diagnosing acute UTIs. ${ }^{2}$ The findings of Bent $e t$ al reinforce these guidelines. Based on their analysis, they conclude that women who present with $\geq 1$ symptom of UTI (dysuria, frequency, haematuria, and back pain) have a high probability of infection (approximately 50\%), and that combinations of symptoms can substantially increase the likelihood of UTI, effectively ruling in the disease based on the history alone. However, they caution that the history and physical examination cannot reliably rule out UTI in women who present with urinary symptoms. Even with a combination of symptoms (absence of dysuria, absence of back pain, and a history of vaginal discharge or vaginal irritation) and 1 sign (vaginal discharge) and a negative dipstick result, the probability of UTI is rarely $<20 \%$. Therefore, a urine culture and pelvic examination should be considered in patients who present with some symptoms of UTI, but mostly negative history and physical examination findings.

Confidence in the findings of this review is strengthened by the thoughtfully constructed methods and well executed analysis. Unfortunately, the authors may have limited their findings by searching only 1 database, Medline. Also, some confusion exists between the symptom of back pain and the physical sign of costovertebral angle (CVA) tenderness. CVA tenderness refers to pain elicited from fist percussion over the kidneys, (ie, the area at which the last rib meets the vertebral column). The patient reported symptom of back pain could refer to discomfort over the CVA or at any other area on the back. Clarification of the term "back pain" would enable clinicians to narrow the questions they ask during the patient history, thus improving provider confidence in their diagnosis

M Kay Libbus, RN, DrPH University of Missouri Columbia, Missouri, USA

1 Seltzer VL, Pearse WH, editors. Women's primary health care: office practice and procedures. New York: McGraw-Hill, 1995.

Leiner S. Recursent urinary tract infections in otherwise healthy adult women. Rational strategies for work-up and management. Nurse Pract 1995;20;48,51-2,54-6. 